\title{
Introduction to the special issue "Beliefs in Groups" of Theory and Decision
}

\author{
Franz Dietrich $^{1}$. Wlodek Rabinowicz ${ }^{2}$
}

Published online: 20 June 2018

(C) Springer Science+Business Media, LLC, part of Springer Nature 2018

This symposium in the overlap of philosophy and decision theory is described well by its title "Beliefs in Groups". Each word in the title matters, with one intended ambiguity. The symposium is about beliefs rather than other attitudes such as preferences; these beliefs take the form of probabilities in the first three contributions, binary yes/no beliefs ('judgments') in the fourth contribution, and qualitative probabilities ('probability grades') in the fifth contribution. The beliefs occur in groups, which is ambiguous between beliefs of groups as a whole and beliefs of group members. The five contributions - all of them interesting, we believe-address several aspects of this general theme.

Where contributions address beliefs of group members, the central question is that of belief revision: how should individuals revise their beliefs after learning those of others? This question is of obvious interest in the context of deliberation and exchange of opinions. By contrast, where contributions address beliefs of the group as a whole, the central question is that of aggregation: how should the beliefs of group members be merged into collective beliefs? The two questions are interconnected in many ways. For one, revising one's beliefs may take the form of aggregating them with learnt beliefs of others - for instance through averaging probability assignments, something analysed in depth in the first three contributions. This approach reduces revision to aggregation. A converse reduction is also imaginable, though not common. One might argue that the right aggregate beliefs are those beliefs which would emerge as consensus beliefs through suitable deliberation and belief revision by the group members, be it in one revision round, finitely many revisions rounds, or a converging infinite

\footnotetext{
$凶$ Franz Dietrich fd@franzdietrich.net

1 Paris School of Economics and CNRS, Paris, France

2 Department of Philosophy, Lund University, Lund, Sweden
} 
sequence of revision rounds. However, whether or not one is ready to reduce revision to aggregation or vice versa, the two phenomena are definitely complementary in an obvious sense: deliberation and belief revision by group members is often the first step, which (when it does not result in consensus) may be followed by an aggregation of the post-deliberation beliefs. All this emphasizes the importance of studying both phenomena in connection to one another. Doing this is the purpose of this symposium.

Let us say a few introductory words about each contribution, without anticipating too much of the secrets and surprises.

Richard Bradley's "Learning from others: conditioning versus averaging”. Suppose it is autumn 2106, just before the United States presidential election. An expert tells you the probability he or she attaches to a victory by the republican candidate Donald Trump, and this subjective probability exceeds yours. How should you revise yours? The simplest proposal is to adopt some weighted average between your and his probability. Bradley asks under what conditions this revision policy can be given a Bayesian rationalization: when does such averaging yield the same new probability as Bayesian conditionalization upon the learnt proposition, i.e., the proposition that the expert assigns such-and-such probability to Trump's victory? The question is not fully new, but Bradley has novel results to offer and sheds new light onto the question, demonstrating just how rare and stylised the scenario of compatibility between revision-by-averaging and Bayesianism is. After reading Bradley's piece, readers will get the sense that Bayesianism is essentially incompatible with revision by averaging. The next two contributions will rehabilitate the rationality of averaging from rather different perspectives.

Denis Bonnay's and Mikaël Cozic's "Weighted Averaging, Jeffrey Conditioning and Invariance". Suppose in the above thought experiment you revise your belief in Trump's victory by adopting a (weighted) average between your and the expert's subjective probabilities. This can only be part of the story, because you still need to revise the rest of your subjective probability function, including your subjective probability of events related to the election outcome, such as the events of a war with North Korea and of a collapse of the international alliance against global warming. As the authors point out, Jeffrey revision is a natural option: after having revised your probability of Trump's victory, you can Jeffrey revise your probability function given this new probability of Trump's victory. In other words, after having revised the probability that Trump wins, you revise the rest of your probability function in such a way as to preserve all probabilities conditional on Trump winning or conditional on him losing, following the principles of rigidity or conservativeness (e.g., Jeffrey 1957 and Dietrich et al. 2016). So revision through averaging is not a rival of Jeffrey revision (and hence of its special case, Bayesian revision), but the other side of the same coin: averaging can regulate revision on those propositions for which expert probabilities are learnt, and then Jeffrey revision can regulate revision on all other propositions. The authors' central mathematical contribution is to show that the two revision policies of averaging and Jeffrey revision, as different as they may seem at first, follow from the same structural principle, an "invariance principle" defined in their contribution. That principle-if accepted-therefore provides foundations for the above-sketched two-stage procedure for revising beliefs in the face of testimonial evidence. 
Jan-Willem Romeijn's and Olivier Roy's “All Agreed: Aumann meets DeGroot”. While Bradley's and Bonnay-Cozic's contributions consider the asymmetric scenario in which one agent learns someone else's probabilistic belief about a proposition and then revises his or her own belief, Romeijn and Roy investigate the symmetric scenario in which two agents exchange their beliefs about the proposition and then each revise their beliefs. Another difference is that the agents do not stop there: they afterwards share their new beliefs and revise again, and so on. This sort of iterated belief revision has already been addressed, yet in two very different ways and by two largely disconnected bodies of literature. On the one hand, DeGroot (1974), Lehrer and Wagner (1981) and followers assume that in each round each agent revises his or her belief in the proposition by averaging it with the learnt belief of the other agent; under idealised conditions, this process leads in the limit to equality between the agents' beliefs about the proposition, i.e., to consensus. On the other hand, an orthodox Bayesian literature started by Aumann (1976) and Geanakoplos and Polemarchakis (1982) takes agents to perform ordinary Bayesian revision in each round; under idealised conditions, this process leads in the limit again to equality between the beliefs in the proposition, and moreover to common knowledge of these beliefs. The authors unconventionally combine the two approaches by asking whether the revision policy of one approach (averaging) can be rationalized in terms of the revision policy of the other (Bayesianism) with common knowledge of beliefs in the limit. If a Bayesian rationalization of repeated averaging-based belief revision has not yet been tackled before, it is perhaps because no-one dared to hope that a revision policy as simple and non-Bayesian as averaging could, in the limit, achieve a result as noble and Bayesian as commonly known identical beliefs. As the authors demonstrate, the hope is justified and sometimes comes true.

Marcel Heidemann's “Judgment Aggregation and Minimal Change - A Model of Consensus Formation by Belief Revision”. This contribution considers binary yes/no beliefs ('judgments') rather than probabilistic beliefs, and focuses not just on revision, but also on aggregation ('judgment aggregation'). While judgment aggregation theory usually interprets aggregate judgments as compromises between diverging individual judgments, Heidemann assumes that individuals revise their initial judgments, taking over the aggregate judgments. So, aggregate judgments play a double-role: they are group judgments as well as revised individual judgments. According to Heidemann, this double-role requires a different approach to judgment aggregation: an approach that imposes different axioms on aggregation rules, and does not result in standard rules such as majority rule, quota rules, premise-based rules, distance-based rules, or scoring rules. Heidemann wants that individuals can adopt the collective judgments as easily as possible through revising their initial judgments. Formally, collective judgments should arise through minimal revision of individual judgments. This new judgment-aggregation paradigm is carried out in a sophisticated formal analysis, inspired by AGM belief revision theory (Alchourron et al. 1985). The results should interest judgment-aggregation theorists as much as belief-revision theorists.

Aidan Lyon's and Michael Morreau's "The Wisdom of Collective Grading and the Effects of Epistemic and Semantic Diversity”. This contribution focuses on aggregation of probabilistic beliefs within a group, yet assuming these beliefs are expressed in a qualitative fashion - based on a given language of grades, for instance one that allows 
for three possible grades, "likely", "a toss-up", and "unlikely". Grades are interpreted as corresponding to probability intervals. The precise interpretation of grades (the lower and upper boundaries of the intervals) might be agreed upon within the group or it might differ across group members. Thus, there might be semantic consensus or semantic diversity. Semantic consensus can often be hard to achieve; just think about the grading systems used to evaluate research grants. But is semantic consensus actually a goal that we should strive for? Suppose the group aims to choose, out of $n$ events, a small number $k$ of most probable events. Given the authors' assumptions about the expertise of group members, it would be epistemically ideal to solve this task by directly averaging the group members' (unknown) precise subjective probabilities of the events. What are the prospects of approximating this ideal through aggregating the imprecise grades given by the members? Will these prospects be better with semantic consensus or with semantic diversity? Lyon and Morreau answer this question using computer simulation. Surprisingly, it turns out that semantic diversity, while it hinders communication, still on the whole offers better prospects, as long as the group has relatively few grades at its disposal. What drives this result? Allowing different individuals to interpret grades differently (semantic diversity) increases the total number of distinctions that can be drawn by combining grades of different individuals. That is, under semantic diversity the aggregation of grades produces a more fine-grained probability ranking of the events in question.

\section{Compliance with ethical standards}

Funding Funding was provided by Agence Nationale de la Recherche (ANR) and Deutsche Forschungsgemeinschaft (DFG) (ColAForm).

\section{References}

Alchourron, C. E., Gardenfors, P., \& Makinson, D. (1985). On the logic of theory change: Partial meet contraction and revision functions. The Journal of Symbolic Logic, 50(2), 510-530.

Aumann, R. J. (1976). Agreeing to disagree. Annals of Statistics, 4(6), 1236-1239.

DeGroot, M. (1974). Reaching a consensus. Journal of the American Statistical Association, 69, 118-121.

Dietrich, F., List, C., \& Bradley, R. (2016). Belief revision generalized: A joint characterization of Bayes's and Jeffrey's rules. Journal of Economic Theory, 162, 352-371.

Geanakoplos, J. D., \& Polemarchakis, H. M. (1982). We cannot disagree forever. Journal of Economic Theory, 28, 192-200.

Jeffrey, R. (1957). Contributions to the theory of inductive probability. Ph.D. Thesis, Princeton University Lehrer, K., \& Wagner, C. (1981). Rational consensus in science and society: A philosophical and mathematical study. Berlin: Springer. 